\title{
Motorized Spiral Enteroscopy for Occult Bleeding
}

\author{
Laura Mans $^{\mathrm{a}}$ Marianna Arvanitakis ${ }^{\mathrm{a}}$ Horst Neuhaus $^{\mathrm{b}}$ Jacques Devière ${ }^{\mathrm{a}}$ \\ a Department of Gastroenterology, Hepatopancreatology, and Digestive Oncology, Hôpital Erasme, Université \\ Libre de Bruxelles, Brussels, Belgium; ${ }^{b}$ Department of Internal Medicine, Evangelisches Krankenhaus Düsseldorf, \\ Düsseldorf, Germany
}

\begin{abstract}
Keywords
Motorized spiral enteroscopy $\cdot$ Occult bleeding $\cdot$ Small bowel disorders
\end{abstract}

\begin{abstract}
Small-bowel disorders remain a diagnostic and therapeutic challenge due to intestinal length. Their management was revolutionized by the emergence of deep enteroscopy. In comparison with video capsule endoscopy, deep enteroscopy allows diagnostic and therapeutic interventions. Spiral enteroscopy (Spirus Medical Endo-Ease Overtube) achieves progression by gentle push and manual clockwise rotation inducing pleating of the small bowel on the endoscope. It has the advantage of shorter examination time and more stability during withdrawal but requires two operators. The Novel Motorized Spiral Enteroscope (Olympus Corp.) incorporates a user-controlled motor contained in the handle of the endoscope. This would offer the possibility to accelerate the procedure, facilitate insertion, and simplify the technique with a single operator.

(c) 2018 S. Karger AG, Basel
\end{abstract}

\section{Background}

Small-bowel endoscopic evaluation was improved by the introduction of video capsule endoscopy (VCE). It permits the visualization of the entire small intestine but is limited by the inability to take biopsy specimens and to perform therapeutic procedures [1-3]. Small-bowel disorders remain a diagnostic and therapeutic challenge due to intestinal length, which makes looping much more difficult to avoid during push enteroscopy $[1,3]$. The introduction of deep enteroscopy, including double-balloon enteroscopy (DBE), single-balloon enteroscopy (SBE), and spiral enteroscopy (SE) could be considered an alternative or complementary procedure with both diagnostic and therapeutic possibilities. Deep enteroscopy, using SBE and DBE devices, are the procedures of choice used in Europe. DBE is the most studied and validated deep enteroscopy technique [3]. SE (Spirus Medical Endo-Ease Overtube) requires a spiral overtube. The progression is made by gentle push and manual clockwise rotation inducing pleating of the small bowel on the endoscope [4]. This device has the advantage of shorter examination time and more stability of withdrawal but requires two operators [2-5]. The comparison of deep enteroscopy techniques is difficult and each of them has its strengths and weaknesses [4]. A recent review and meta-analysis comparing balloon enteroscopy versus SE resulted in similar outcomes for diagnostic and therapeutic rates and also for depth insertion, but SE seems to be shorter in procedural time [2]. The Novel Motorized Spiral Enteroscope (NMSE, Olympus Corp.) is a new technology with an incorporated user-controlled motor contained in the

Live case presented during the 35th GEEW 2017, Brussels, Belgium.

\section{KARGER}

(c) 2018 S. Karger AG, Basel

E-Mail karger@karger.com

www.karger.com/ddi
Laura Mans

Department of Gastroenterology, Hepatopancreatology, and Digestive Oncology

Hôpital Erasme, Université Libre de Bruxelles

Route de Lennik 808, BE-1070 Brussels (Belgium)

E-Mail laura.mans@erasme.ulb.ac.be 

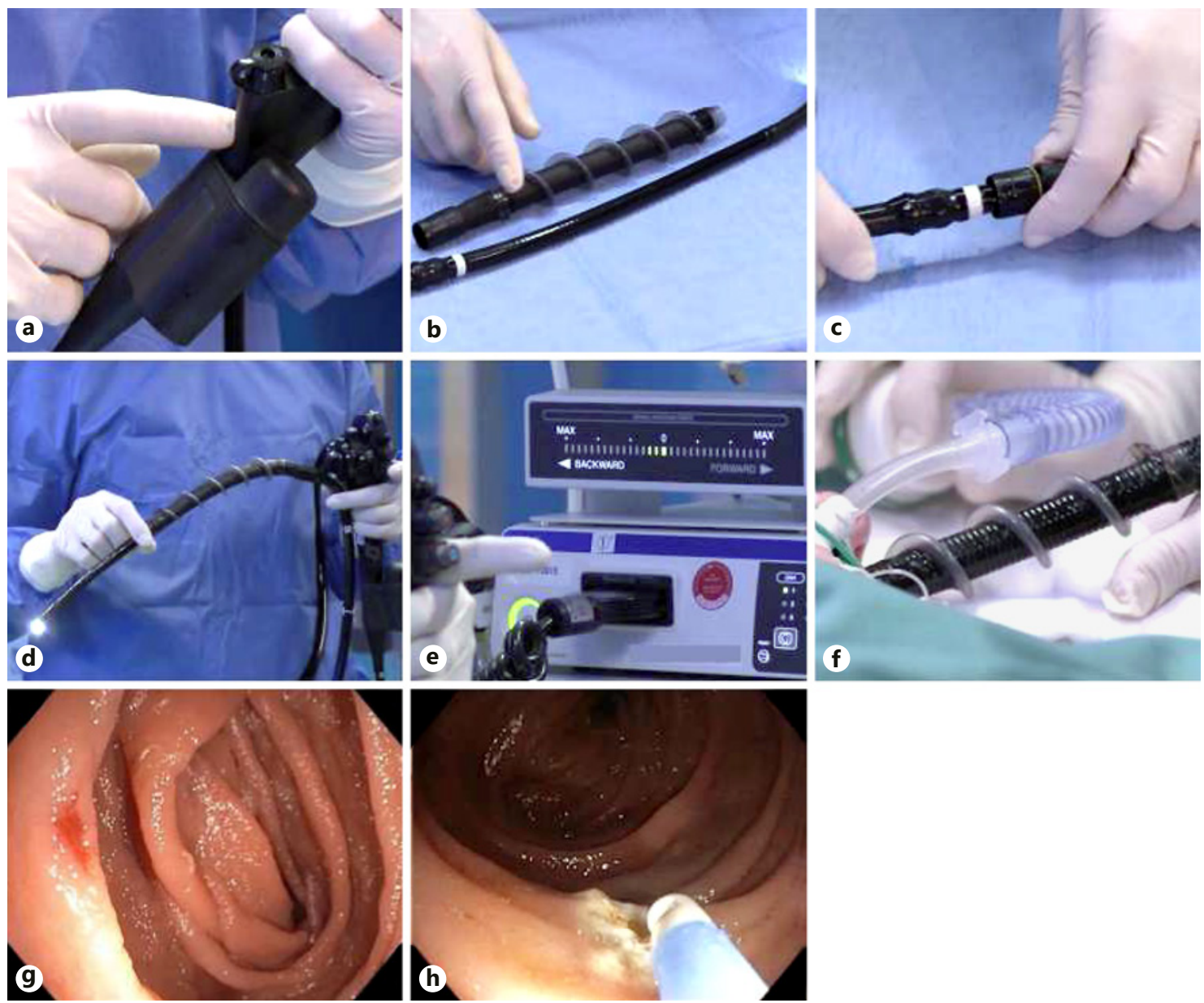

Fig. 1. a Integrated electric motor of the novel enteroscope. b Disposable short spiral overtube and rotation coupler of the insertion tube. $\mathbf{c}$ The spiral overtube is connected to the rotation coupler. $\mathbf{d}$ Prototype spiral enteroscope ready for use. e Spiral rotation force indicator. $\mathbf{f}$ Insertion of spiral portion of the endoscope. $\mathbf{g}$ Detection of arteriovenous malformation of the jejunum during spiral enteroscopy. $\mathbf{h}$ Treatment of arteriovenous [6].

handle of the endoscope. This would offer the possibility to accelerate the procedure, facilitate insertion, and simplify the technique with a single operator. Recently, a clinical case report showed the first use of this device for successful treatment of angiodysplasia initially seen by VCE, with no adverse events [6]. Interim analysis of a recent pilot study showed a high diagnostic yield with few adverse events and no severe adverse events.

\section{Materials and Methods}

NMSE (Olympus Corp.) (Fig. 1) is composed of three subsystems: (1) a reusable endoscope with integrated motor permitting the rotation of a (2) short spiral overtube placed on the insertion tube portion of the endoscope, and (3) a motor control unit with a foot pedal and visual force gauge. The reusable endoscope is a dedicated enteroscope of $168 \mathrm{~cm}$ insertion section working length with an outer diameter of $12.8 \mathrm{~mm}$ and a channel inner diameter of $3.2 \mathrm{~mm}$.

The foot pedal activates the drive motor located in the endoscope handle, which controls the direction and speed of rotation of a coupler located in the middle of the insertion tube of the endoscope. The rotation rate is maximum $30 \mathrm{rpm}$ and depends on the pressure applied to the foot pedal. The bowel is pleated or unpleated on the insertion tube of the endoscope depending on the rotation in clockwise or counter-clockwise direction, respectively. During the examination, the visual force gauge allows the operator to monitor the direction of rotation and the resistance applied to the spiral overtube. After reaching the depth of insertion required for patient treatment or diagnosis, the endoscope will be withdrawn using motorized counter-clockwise spiral rotation, also controlled by the foot pedal. 


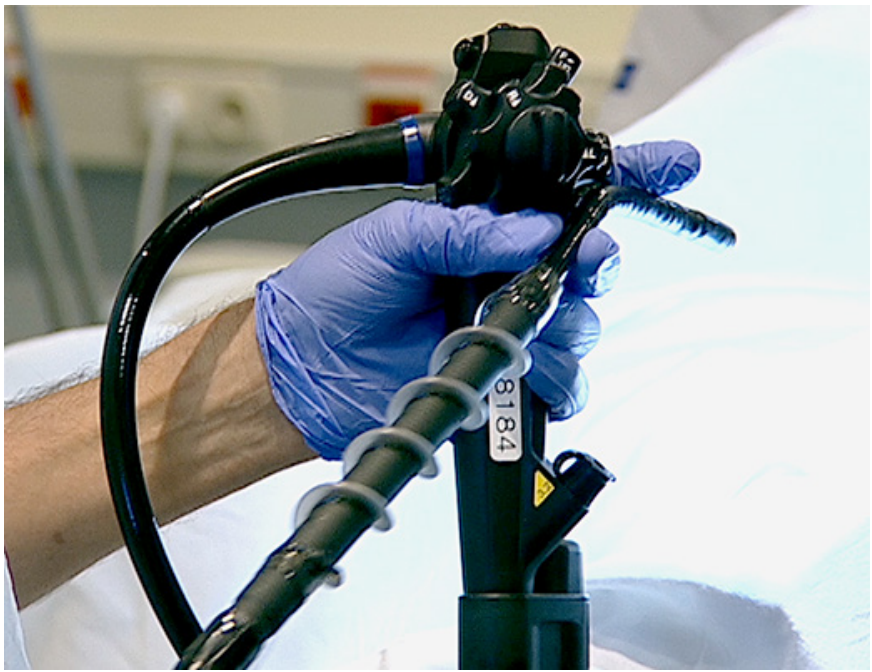

Video 1. Novel motorized spiral enteroscopy (NMSE, Olympus Corp.) - clinical case.

We describe the case of a 71-year-old man with iron deficiency anemia (hemoglobin at $7.2 \mathrm{~g} / \mathrm{dL}$ ) and positive fecal occult blood test. The patient is treated for atrial fibrillation with anticoagulants. Esophagogastroduodenoscopy and colonoscopy were normal. VCE showed two angiodysplasias in the proximal and distal jejunum. SBE allowed the coagulation of angiodysplasias in the proximal jejunum, but progression was limited. Unfortunately, the patient experienced a recurrence of anemia. He was scheduled for NMSE.

\section{Results and Discussion}

The procedure was performed under general anesthesia and in left lateral decubitus position. The device was inserted and advanced with the assistance of motorized clockwise spiral rotation. The maximal depth of insertion was $2 \mathrm{~m}$ in the small bowel (estimated by fold-counting method [7]) with a time of progression of $30 \mathrm{~min}$. Two angiodysplasias were visualized during the progression and treated by argon plasma coagulation. The withdrawal time was $20 \mathrm{~min}$. The patient was kept under observation for the first $24 \mathrm{~h}$, the immediate follow-up was uneventful, and the patient was discharged at day 1 . In case of anemia recurrence, a retrograde NMSE should be discussed.

\section{Conclusion}

NMSE is an alternative technique for diagnostic and therapeutic deep enteroscopy. Further studies and particularly large controlled trials are needed to determine the role of this device.

\section{Acknowledgments}

The authors would like to thank the teamwork during the 35th GEEW 2017 and specifically: medical doctors, foreign experts, nurses, and administrative assistants.

\section{Statement of Ethics}

The patient provided written informed consent to undergo this procedure and the study was approved by our Ethics Committee.

\section{Disclosure Statement}

The authors have no conflicts of interest to disclose.

\section{Funding Sources}

The device for this study was provided by Olympus Corp.

\section{References}

Motorized Spiral Enteroscopy for Occult Bleeding
1 ASGE Standards of Practice Committee, Khashab MA, Pasha SF, Muthusamy VR, et al: The role of deep enteroscopy in the management of small-bowel disorders. Gastrointest Endosc 2015;82:600-607.

2 Baniya R, Upadhya S, Subedi SC, et al: Balloon enteroscopy versus spiral enteroscopy for small-bowel disorders: a systematic review and meta-analysis. Gastrointest Endosc 2017;S0016-5107:32063-32061.

3 Rahmi G, Samaha E, Vahedi K, et al: Multicenter comparison of double-balloon enteroscopy and spiral enteroscopy. J Gastroenterol Hepatol 2013;28:992-998.
4 Akerman PA: Spiral enteroscopy versus double-balloon enteroscopy: choosing the right tool for the job. Gastrointest Endosc 2013;77: 252-254.

5 Akerman PA, DeMarco DC, Pangtay J, et al: A novel motorized spiral enteroscope can advance rapidly, safely and deeply into the small bowel. Gastrointest Endosc 2011;73:AB446.

6 Neuhaus H, Beyna T, Schneider M, et al: Novel motorized spiral enteroscopy: first clinical case. Gastrointest Endosc 2016;83:AB637.

$7 \mathrm{Kim}$ J: Training in endoscopy: enteroscopy. Clin Endosc 2017;50:328-333. 